\title{
Dealing with daily challenges in dementia (deal-id study): effectiveness of the experience sampling method intervention 'Partner in Sight' for spousal caregivers of people with dementia: design of a randomized controlled trial
}

Rosalia J. M. van Knippenberg ${ }^{1}$, Marjolein E. de Vugt ${ }^{*}$, Rudolf W. Ponds ${ }^{1}$, Inez Myin-Germeys ${ }^{2}$ and Frans R. J. Verhey ${ }^{1}$

\begin{abstract}
Background: There is an urgent need for psychosocial interventions that effectively support dementia caregivers in daily life. The Experience Sampling Methodology (ESM) offers the possibility to provide a more dynamic view of caregiver functioning. ESM-derived feedback may help to redirect caregivers' behavior towards situations that elicit positive emotions and to increase their feelings of competence in the caretaking process. This paper presents the design of a study that evaluates the process characteristics and effects of the ESM-based intervention 'Partner in Sight'.

Methods/design: A randomized controlled trial with 90 spousal caregivers of people with dementia will be conducted. Participants will be randomly assigned to the experimental (6-week ESM intervention including feedback), pseudo-experimental (6-week ESM intervention without feedback), or control group (care as usual). Assessments will be performed pre- and post-intervention and at 2-, and 6-month follow-up. Main outcomes will be sense of competence, perceived control, momentary positive affect, and psychological complaints (depressive symptoms, perceived stress, anxiety, momentary negative affect). In addition to the effect evaluation, a process and economic evaluation will be conducted to investigate the credibility and generalizability of the intervention, and its cost-effectiveness.

Discussion: The potential effects of the ESM intervention may help caregivers to endure their care responsibilities and prevent them from becoming overburdened. This is the first ESM intervention for caregivers of people with dementia. The results of this study, therefore, provide a valuable contribution to the growing knowledge on m-health interventions for dementia caregivers.
\end{abstract}

Trial registration: Dutch Trial Register NTR4847; date registered Oct 9, 2014.

Keywords: Dementia, Caregiver, Support, ESM intervention, RCT

\footnotetext{
* Correspondence: m.devugt@maastrichtuniversity.nl

${ }^{1}$ Department of Psychiatry and Neuropsychology and Alzheimer Centre

Limburg, School for Mental Health and Neurosciences, Maastricht University,

Maastricht, The Netherlands

Full list of author information is available at the end of the article
} 


\section{Background}

Caregivers of people with dementia (PwD) are at great risk of becoming overburdened and of developing psychological and physical symptoms during the caretaking process [1]. This calls for psychosocial interventions that effectively support caregivers of PwD in daily life and help them handling their care responsibilities.

Various psycho-social interventions have been developed in recent years for caregivers of $\mathrm{PwD}$, including psycho-education, emotional support, practical assistance, cognitive-behavioral therapy, and multi-component interventions [2]. Overall, research has shown significant but small effects of current interventions on caregiver outcomes. A common feature in these studies is that outcome measures include retrospective self-assessments that are highly susceptible to emotional and cognitive biases [3]. Moreover, retrospective methods do not provide information about fluctuations in subjective experiences over time and across situations that caregivers of PwD may face due to the continually changing care demands.

The Experience Sampling Methodology (ESM) is an innovative approach in assessing subjective experiences in real-time within the flow of daily life. ESM consists of a structured diary method in which repeated self-assessments are electronically recorded the moment they occur, in their natural setting $[4,5]$. ESM offers the possibility to provide a more accurate and detailed view of caregiver functioning, since it enables daily fluctuations in subjective experiences to be explored and it minimizes retrospective recall biases $[6,7]$. Therefore, ESM might be a valuable addition to standard retrospective methods, particularly in older populations with an increased incidence of memory deficits [8].

Recently, there has been growing interest in adapting ESM to clinical practice. By using modern technology, such as personal digital assistants (PDAs) and apps, momentary data are immediately available to both caregivers and professionals. This creates the opportunity to develop ESM interventions that provide explicit, visualized feedback on implicit dynamic patterns of feelings, experiences, and behavior $[9,10]$. Receiving feedback on behavior can result in emotional and behavioral change, something already known from the field of behavioral therapy $[11,12]$. The feedback may help caregivers redirect their behavior towards situations that are conducive to positive emotional experiences. In this way, ESM offers the opportunity to actively involve caregivers in their own empowering process and to provide more personally tailored support [13]. Both these aspects have been demonstrated to be essential in effective psychosocial interventions $[14,15]$.

A focus on positive experiences facilitates a more positive interaction between the caregiver and the PwD and increases positive emotions in both the parties [16]. According to the 'broaden-and-build theory', positive emotions elevate the ability to cope with stressful situations and might consequently help to increase caregivers' feelings of being capable of caring for the PwD $[17,18]$. Positive emotions could thus be an important target in caregiver support interventions, increasing caregiver well-being and reducing long-term negative impacts, such as stress and burden $[19,20]$.

In a recent study, ESM-derived feedback on positive affect was provided to persons with depression during a 6week intervention period. Its results showed that personalized feedback increased self-awareness and resulted in a significant decline in depressive symptoms [9]. In another ESM study, in which depressed individuals collected ESM data for scientific purposes but without receiving feedback, some participants reported that responding to the ESM questionnaires had already 'helped them' and enhanced their awareness of their daily functioning [21]. So far, few studies have applied ESM in caregivers of PwD in the context of research [22, 23]. Recent evidence suggests that ESM is a feasible method for use with this often elderly and vulnerable population (van Knippenberg et al.: Dealing with daily challenges in dementia (Deal-id study): an innovative approach to assess caregiver functioning in the flow of daily life, submitted). However, to date, no ESM interventions have been developed to support caregivers of PwD in dealing with daily challenges associated with dementia. This paper describes the design of a randomized controlled trial (RCT) to evaluate the effects of the ESM-based intervention program 'Partner in Sight' for spousal caregivers of PwD.

\section{Study aims}

The specific objectives of the current study are:

1. Process evaluation to investigate the internal and external validity of the intervention based on sampling quality (recruitment, randomization, and reach) and intervention quality (relevance, feasibility, and performance according to protocol). The process evaluation will be conducted prior to the effect evaluation in order to provide essential information about credibility and generalizability [24].

2. Effect evaluation to assess whether 'Partner in Sight' is superior to a pseudo-intervention and control group in terms of producing a clinically significant increase in subjective well-being, as proven by an increase in caregivers' sense of competence, perceived control, momentary positive affect, and a decrease in psychological complaints (depression, anxiety, stress, and momentary negative affect). A follow-up evaluation will be conducted to examine whether the effects have lasted two and six months after the intervention.

3. Economic evaluation to assess the cost-effectiveness of 'Partner in Sight' by estimating the impact of the intervention on resource use, costs, and health outcomes. 


\section{Methods and design}

The study is a randomized controlled trial with three treatment arms. The experimental condition in which caregivers participate in the ESM-intervention 'Partner in Sight' (ESM data collection including feedback) will be compared with a pseudo-experimental condition (ESM data collection without feedback) and a control group (care as usual). Data will be collected pre- and post-intervention and at two- and six-months follow-up (Fig. 1).

\section{Study population}

The study population will consist of spousal caregivers of community dwelling people with all subtypes and stages of dementia. No age limit will be applied. Participants will be recruited in memory clinics (Maastricht University Medical Center $+(\mathrm{MUMC}+)$, Atrium Medical Center Parkstad), ambulatory mental health care institutions (VirenzeRIAGG Maastricht, Lionarons GGZ), dementia day care centers (Sevagram, NOVIzorg, Orbis Glana, Proteion, care farm Ransdalerveld), and caregiver support services (Hulp bij Dementie, Steunpunt Mantelzorg) in the southern region of the Netherlands, and via the website of the Dutch Alzheimer Society. The clinician or care counselor who is involved in the treatment of the PwD will approach caregivers to participate in the study. Subsequently, potential participants will be contacted and screened by the researcher to make sure that they fully meet the following inclusion criteria: (1) being a spousal caregiver of a person with a diagnosis of dementia; (2) sharing a household with the PwD; and (3) informed consent obtained. Exclusion criteria will be: insufficient cognitive abilities to engage in ESM; being overburdened or having severe health problems based on clinical judgment of a knowledgeable practitioner; taking care for a PwD caused by Human Immunodeficiency Virus (HIV), acquired brain injury, Down syndrome, chorea related to Huntington's disease or alcohol abuse.

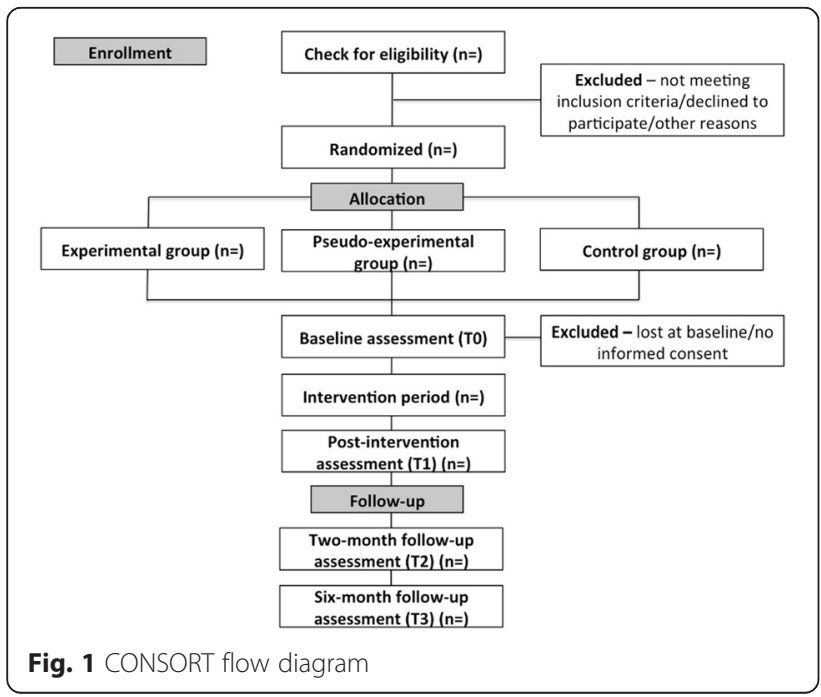

\section{Randomization}

Caregivers will be randomly assigned to the experimental group, pseudo-experimental group, or control group. Randomization will be computer-generated and conducted by an independent statistician. Block randomization will be performed to diminish the risk of an unbalanced assignment to the three treatment arms. Randomly permuted blocks with variable block sizes (3, 6, and 9) will be used, by which the block size and specific order will be chosen randomly at the beginning of each block. This reduces the risk of predicting group assignment and keeps research staff blind to the randomization process. The design of this study is single-blind. An independent research assistant, who is being blinded to the treatment allocation, will conduct the baseline, post-intervention and follow-up assessments, and will be asked to evaluate success of blinding and reasons for possible unmasking on the Case Record Form.

\section{ESM procedure}

ESM will be carried out using the PsyMate, an electronic touchscreen device that is specifically designed to monitor experiences and behavior in daily life and that offers the possibility to provide immediate ESM-derived feedback (www.psymate.eu). The PsyMate has been extensively studied and refined in several studies concerning psychiatric populations (e.g. psychosis and depression) [10, 13]. In a recent ESM study with spousal caregivers of PwD, the PsyMate was considered to be a user-friendly and easily accessible device (van Knippenberg et al.: Dealing with daily challenges in dementia (Deal-id study): an innovative approach to assess caregiver functioning in the flow of daily life, submitted).

The PsyMate will be programmed to generate ten beeps (sound and vibration) per day at random intervals between 7:30 AM and 10:30 PM. ESM will be used as an assessment tool during the baseline assessment (three consecutive days $=30$ beeps in total) and the post assessment (three consecutive days $=30$ beeps in total) to evaluate the effectiveness of the intervention. Furthermore, ESM will be used as an intervention tool during the 6-week intervention period (three consecutive days per week $=10 \times 3 \times 6=180$ beeps in total). In order to include different days of the week, the PsyMate will beep alternately on Friday, Saturday, and Sunday, or Tuesday, Wednesday, and Thursday during the intervention period.

After each beep, caregivers will be asked to complete a questionnaire presented on the screen of the PsyMate, including current affect (four positive affect and eight negative affect items), self-esteem (four items), physical well-being (four items), as well as current context and activities (daily life activities, social company, location and events). At the end of each questionnaire, caregivers will have to indicate whether the beep disturbed them. 
Answering all questions will take approximately three minutes per beep (van Knippenberg et al.: Dealing with daily challenges in dementia (Deal-id study): an innovative approach to assess caregiver functioning in the flow of daily life, submitted). Additionally, the PsyMate will be programmed to generate a morning and evening questionnaire at the beginning and end of each day. The morning questionnaire consists of six items regarding their sleep quality during the previous night and their current level of energy. The evening questionnaire contains twenty-five items concerning the caregivers' daily sense of well-being and competence, and neuropsychiatric symptoms in the PwD during that day. Responses will be rated on 7-point Likert scales (ranging from 1 'not at all' to 7 'very much'), bipolar scales (ranging from -3 'very unpleasant/very unimportant' to +3 'very pleasant/very important'), a Visual Analogue Scale (ranging from 0 'worst imaginable health' to 100 'best imaginable health'), and box-checking formats. Responses cannot be corrected afterwards. An overview of the ESM items with corresponding response choices and concepts is presented in the Appendix. The specific items of the ESM questionnaires were developed and selected according to information available from previous ESM studies [25, 26], knowledge about the experiences and situations that caregivers of PwD could be expected to encounter in daily life, and the guidelines for item development created by ESM experts [27]. Moreover, the ESM questionnaires were recently tested in a feasibility study that yielded positive results (van Knippenberg et al.: Dealing with daily challenges in dementia (Deal-id study): an innovative approach to assess caregiver functioning in the flow of daily life, submitted).

\section{Study procedure}

\section{Baseline assessment (TO)}

After the randomization procedure, a baseline assessment (T0) will take place in the caregiver's home or at the MUMC+ according to their preference. Participants will be asked by the research assistant to sign the informed consent before continuation of the study procedure. Next, a demographical interview will be conducted to assess caregiver and care recipient characteristics. Additionally, caregivers will be asked to participate in a 3-day ESM baseline measurement, starting the day after the baseline assessment. A 30-min briefing will be provided during the baseline assessment to ensure that they fully understand the procedure and how to operate the PsyMate. A demo questionnaire will be presented to familiarize caregivers with the device and a leaflet containing all relevant information regarding the use of the device will be handed out. Finally, the caregivers will be asked to complete a number of retrospective questionnaires at their own convenience as part of the effect evaluation.

\section{Intervention period}

Experimental group Caregivers in the experimental group will participate in the 6-week intervention 'Partner in Sight' and collect ESM data for three consecutive days a week. Every two weeks they will receive ESM-derived feedback in a face-to-face session with a coach in their home or at the MUMC+ according to their preference. This sums up to a total of three feedback sessions. The aim of the feedback sessions is to provide an overview of the caregiver's everyday functioning, including their mood (i.e. levels of positive affect), daily life activities, and social interactions. The focus will be on positive rather than negative emotional experiences and how these relate to specific daily contexts. In this way, we try stimulate caregivers to redirect their behavior towards situations that elicit positive emotions.

At the beginning of each feedback session average levels of positive affect experienced during the past two weeks will be presented. Subsequently, a feedback module on daily activities and/or social interactions in daily life will be discussed with the caregiver. Feedback modules are based on an existing ESM intervention, which has proven to be effective in people with depression [9]. During the first feedback session the module 'daily activities' will be discussed with the caregiver. The data may, for example, illustrate that positive emotions increase during moments of active relaxation, while the caregiver is actually spending the least amount of time on active relaxation activities. The coach will stimulate caregivers to think about the findings and to implement new insights into their daily lives. In the second feedback session the module 'social interactions in daily life' will be added. A caregiver might state, for example, that he prefers to spend his time alone, without any company. The feedback, however, may show that positive emotions are experienced particularly while being in company instead of when being alone. This finding might induce an increase in social interactions, which in turn may lead to increased positive emotions. The third session will combine both modules. At the end of each session the weekly progress in levels of positive affect yielded by daily activities and/or social interactions will be evaluated. A more detailed description of the feedback modules is presented in Table 1.

The coach will present all feedback verbally and graphically (in clear pie charts and bar graphs) to the caregiver according to a standardized protocol. Before the start of the study all coaches will receive training with clear instructions on how to provide feedback. After each session both the caregiver as well as the involved clinician or care counselor will be provided with a written copy of the feedback.

At the end of each feedback session there will be a debriefing concerning the use of the PsyMate, e.g. difficulties operating the device, technical problems, items that are unclear, and reasons for missing measurements. Furthermore, the participant will be reminded and encouraged 
Table 1 Feedback modules of the intervention 'Partner in Sight'

\begin{tabular}{|c|c|c|}
\hline Session & Feedback Module & Description \\
\hline \multirow[t]{6}{*}{1} & 1 Daily activities & $\begin{array}{l}\text { - Pie chart of the average time spent on different activities (e.g. caring for partner, } \\
\text { household, active relaxation, passive relaxation, resting, self care) during the past } \\
\text { two weeks }\end{array}$ \\
\hline & & $\begin{array}{l}\text { - Graph demonstrating the relationship between different kind of activities and } \\
\text { experienced levels of positive affect }\end{array}$ \\
\hline & & $\begin{array}{l}\text { - Pie chart of the average time spent on active relaxation in the presence and } \\
\text { absence of the PwD }\end{array}$ \\
\hline & & $\begin{array}{l}\text { - Graph showing experienced levels of positive affect during active relaxation } \\
\text { in the presence and absence of the PwD }\end{array}$ \\
\hline & & $\begin{array}{l}\text { - Pie chart of the average time spent on passive relaxation in the presence and } \\
\text { absence of the PwD }\end{array}$ \\
\hline & & $\begin{array}{l}\text { - Graph showing experienced levels of positive affect during passive relaxation } \\
\text { in the presence and absence of the PwD }\end{array}$ \\
\hline \multirow[t]{3}{*}{2} & $1 \& 2$ Social interactions & Added: \\
\hline & & $\begin{array}{l}\text { - Pie chart of the average time spent in different kinds of company (e.g. partner, } \\
\text { friends, family, colleagues, alone) during the past two weeks }\end{array}$ \\
\hline & & $\begin{array}{l}\text { - Graph demonstrating the relationship between different types of social company } \\
\text { and experienced levels of positive affect }\end{array}$ \\
\hline 3 & $1 \& 2$ & See description above \\
\hline \multirow[t]{4}{*}{$1,2,3$} & General graphs & $\begin{array}{l}\text { - Graph including information on average levels of positive affect during the past } \\
\text { two weeks }\end{array}$ \\
\hline & & $\begin{array}{l}\text { - Graph demonstrating the weekly progress in levels of positive affect during } \\
\text { the course of the intervention }\end{array}$ \\
\hline & & $\begin{array}{l}\text { - Graph demonstrating the weekly progress in levels of positive affect yielded by } \\
\text { daily activities }\end{array}$ \\
\hline & & $\begin{array}{l}\text { - Graph demonstrating the weekly progress in levels of positive affect yielded } \\
\text { by social interactions }\end{array}$ \\
\hline
\end{tabular}

$P w D$ person with dementia

to fill out the PsyMate during the following two weeks as accurately as possible conform the instructions given by the coach. During the third feedback session the coach will additionally conduct an unstructured interview to assess the feasibility of the intervention.

Pseudo-experimental group A pseudo-experimental group is added to the study design to investigate whether the ESM-derived feedback adds value to the use of the PsyMate without receiving any direct feedback. Repeated self-assessments might already increase caregivers' selfawareness and redirect their behavior accordingly [21].

Caregivers in the pseudo-experimental group will participate in a 6-week pseudo-intervention and collect ESM data for three days a week. Every two weeks they will receive a face-to-face session with a coach. However, during these sessions they will not be provided with feedback on their daily recordings. Alternatively, a semi-structured interview on their well-being during the past two weeks will be performed to prevent any effects of different duration of contact with the coach.

At the end of each session caregivers will, similar to the participants in the experimental group, be provided with a debriefing concerning the use of the ESM-device.
Control group Caregivers in the control group will receive care as usual during the 6-week intervention period. Care conditions will differ among caregivers and will be registered carefully at baseline. In general, care as usual includes low-frequent sessions with a clinician from the memory clinic or counselor from a caregiver support service.

\section{Post-intervention assessment (T1)}

After the intervention period a post-intervention assessment will be executed in the caregiver's home or at the MUMC+ according to their preference. As part of the post-intervention assessment, caregivers will be asked to participate in a 3-day ESM post measurement. They will be shortly briefed with respect to the procedure. Afterwards, all participants will be asked to complete a questionnaire concerning their general experiences with the ESM-device during the complete study period. Based on their answers a semi-structured interview will be conducted to discuss the questionnaire. Finally, caregivers will be asked again to complete a number of retrospective questionnaires at their own convenience as part of the effect evaluation. 


\section{Follow-up assessments (T2 \& T3)}

A two- and six-month follow-up assessment will be administered in which participants receive a number of retrospective questionnaires by post and are asked to return them after completion.

\section{Retention}

Participants will be provided with periodic newsletters to inform them about the current status of the study, plans for the next phase, as well as to acknowledge their support.

\section{Instruments}

For an overview of the instruments used during the baseline, post-intervention and follow-up assessments see Table 2 .

\section{Primary outcome measures}

Sense of Competence: caregivers' subjective feelings of competence will be assessed with the Short Sense of Competence Questionnaire (SSCQ), a shortened version of the 27-item Sense of Competence Questionnaire (SSQ) [28]. The SSCQ consists of seven items, rated on a 5-point scale from 1 (agree very strongly) to 5 (disagree very strongly). The items reflect three domains of caregivers' feelings of being capable to care for the PwD: satisfaction with their own performance as a caregiver (2 items), satisfaction with the $\mathrm{PwD}$ as a care recipient (3 items), and consequences of involvement in care for personal life of the caregiver (2 items). A total sum score (range 7-35) will be calculated for each participant. Higher sum scores represent higher levels of sense of competence. The scale displays good content and construct validity in previous research [28].

Table 2 Flowchart of measures used during the assessments

\begin{tabular}{|c|c|c|c|c|c|c|c|}
\hline & \multirow{2}{*}{$\begin{array}{l}\text { Pre-test } \\
T=0\end{array}$} & \multicolumn{3}{|c|}{ Intervention period } & \multicolumn{3}{|c|}{ Post-tests } \\
\hline & & $\overline{\mathrm{FB} 1}$ & FB2 & FB3 & $T=1$ & $\mathrm{~T}=2$ & $\mathrm{~T}=3$ \\
\hline \multicolumn{8}{|l|}{ Primary outcome measures $\mathrm{RCT}$} \\
\hline - Sense of competence: SSCQ & $x$ & & & & $x$ & $x$ & $x$ \\
\hline - Perceived control: PMS & $x$ & & & & $x$ & $x$ & $x$ \\
\hline \multicolumn{8}{|l|}{ Secondary outcome measures RCT } \\
\hline - Depressive symptoms: CES-D & $x$ & & & & $x$ & $x$ & $x$ \\
\hline - Perceived stress: PSS & $x$ & & & & $x$ & $x$ & $x$ \\
\hline - Anxiety symptoms: HADS-A & $x$ & & & & $x$ & $x$ & $x$ \\
\hline \multicolumn{8}{|l|}{ ESM outcome measures RCT } \\
\hline - Momentary positive affect & $x$ & & & & $x$ & & \\
\hline - Momentary negative affect & $x$ & & & & $x$ & & \\
\hline \multicolumn{8}{|l|}{ Additional measures RCT } \\
\hline - Demographic variables & $x$ & & & & & & \\
\hline - Neuropsychiatric symptoms in PwD: NPI-Q & $x$ & & & & $x$ & $x$ & $x$ \\
\hline $\begin{array}{l}\text { - Quality of the relationship: } 4 \text { items of the University of Southern California } \\
\text { Longitudinal Study of Three-Generation Families measures of positive affect }\end{array}$ & $x$ & & & & $x$ & $x$ & $x$ \\
\hline - Coping: UCL & $x$ & & & & $x$ & $x$ & $x$ \\
\hline - Personality: subscale neuroticism of NEO-FFI & $x$ & & & & $x$ & $x$ & $x$ \\
\hline \multicolumn{8}{|l|}{ Outcome measures process evaluation } \\
\hline $\begin{array}{l}\text { - Subjective experiences with intervention: } \\
\text { quantitative questionnaire \& qualitative semi-structured interview }\end{array}$ & & & & $x$ & & & \\
\hline $\begin{array}{l}\text { - Subjective experiences with the use of the ESM device: } \\
\text { quantitative questionnaire }\end{array}$ & & & & & $x$ & & \\
\hline $\begin{array}{l}\text { - Subjective experiences with ESM procedure: } \\
\text { quantitative questionnaire }\end{array}$ & & $x$ & $x$ & $x$ & $x$ & & \\
\hline \multicolumn{8}{|l|}{ Outcome measures economic evaluation } \\
\hline - Resource use: RUD-lite & $x$ & & & & $x$ & $x$ & $x$ \\
\hline - Quality of life: EQ-5D & $x$ & & & & $x$ & $x$ & $x$ \\
\hline
\end{tabular}

$R C T$ randomized controlled trial, SSCQ Short Sense of Competence Questionnaire, PMS Pearlin Mastery Scale, CES-D Center for Epidemiological Studies Depression Scale, PSS Perceived Stress Scale, HADS-A Hospital Anxiety and Depression Scale, Anxiety subscale, PwD person with dementia, NPI-Q Neuropsychiatric Inventory Questionnaire, UCL Utrechtse Coping List, NEO-FFI NEO Five-Factor Inventory, ESM Experience Sampling Methodology, RUD-lite Resource Utilization in Dementia shortened version, EQ-5D EuroQoL-5D 
Perceived control: the extent to which a person perceives him- or herself to be in control of events and on-going situations, also known as mastery, will be measured with the Pearlin Mastery Scale (PMS) [29]. The scale contains seven items with scores varying from 0 (completely agree) to 4 (completely disagree). Items are summed to form a total mastery score (range 0-28), with higher scores indicating greater perceived control. The psychometric properties of the PMS are good according to previous research [30].

\section{Secondary outcome measures}

Depressive symptoms: the Center for Epidemiological Studies Depression Scale (CES-D) will be used to assess depressive symptoms among caregivers [31]. It includes twenty items that rate the frequency of symptoms during the past week. Item scores range from 0 (rarely or none of the time present [less than 1 day]) to 3 (most or all of the time present [5-7 days]). The total sum score ranges from 0 to 60, with higher scores indicating more depressive symptoms. Items depict major components of depressive symptomatology, such as depressed mood, feelings of guilt and worthlessness, feelings of helplessness and hopelessness, psychomotor retardation, loss of appetite, and sleep disturbance. The CES-D has been widely used in research on caregiving and has proven to be sensitive to changes in caregiver depressive symptoms after intervention [14].

Perceived stress: the Perceived Stress Scale (PSS) will be used to measure the degree to which situations in one's life are appraised as stressful [32]. The PSS consists of ten items, rated on a 5-point scale from 0 (never) to 4 (very often), regarding unpredictability, control, and overload. Total sum scores on the PSS range from 0 to 40, with higher scores representing higher levels of stress. Adequate validity and reliability has been demonstrated in previous research [32].

Anxiety symptoms: the 7-item anxiety subscale of the Hospital Anxiety and Depression Scale (HADS) will be employed to assess the severity of anxiety symptoms in caregivers [33]. Item scores range from 0 (not at all) to 3 (a great deal of the time) and will be accumulated to produce a total sum score (range 0-21), with higher scores indicating more anxiety. The HADS has frequently been used in caregivers of PwD and has shown good reliability rates [34].

\section{ESM outcome measures}

Momentary positive and negative affect: the ESM data collected during the 3-day ESM baseline measurement and 3-day ESM post measurement will be used to assess caregivers' momentary positive and negative affect. Positive affect will be defined as the mean score of the items: 'I feel cheerful,' 'I feel relaxed,' 'I feel enthusiastic', and 'I feel satisfied'. Negative affect will be defined as the mean score of the items 'I feel insecure,' 'I feel lonely', 'I feel anxious,' 'I feel irritated', 'I feel down', 'I feel desperate,' 'I feel tensed', and 'I feel confident'. A mean positive and negative affect score will be calculated for each completed beep during the day, with higher scores indicating higher levels of positive and negative affect.

\section{Additional measures}

Demographics: demographic variables, including age, sex, and level of education of both the caregiver and the care recipient, will be assessed during a demographical interview with the caregiver. Furthermore, information about the type, severity, and duration of dementia, caregiver hours of contact with the PwD, caregiver hours of caring for the PwD, and PwD hours spent in a dementia day care setting will be collected. The Clinical Dementia Rating scale (CDR) will be used to stage the severity of the dementia [35]. The CDR score is rated on a 5-point scale: $0=$ 'normal'; 0.5 = 'very mild dementia'; $1=$ 'mild dementia'; 2 = 'moderate dementia'; and 3 ='severe dementia'. The CDR has become widely accepted in the clinical setting as a reliable and valid global assessment measure of dementia [36].

Neuropsychiatric symptoms in the PwD: the Neuropsychiatric Inventory Questionnaire (NPI-Q), a brief form of the Neuropsychiatric Inventory (NPI), will be used to evaluate neuropsychiatric symptoms in the PwD and associated caregiver distress [37]. The NPI-Q evaluates twelve neuropsychiatric domains, including: delusions, hallucinations, agitation/aggression, dysphoria/ depression, anxiety, euphoria, apathy, disinhibition, irritability, aberrant motor behavior, nighttime behavioral disturbances, and appetite and eating abnormalities. For each domain, the caregiver answers a screening question to indicate whether the symptom is present or not. If present, the severity is rated on a scale from 1 (mild) to 3 (severe). Total sum scores range from 0 to 36 . Additionally, a caregiver distress score is rated for each domain on a 6-point scale, ranging from 0 (not emotionally stressful) to 5 (extremely stressful). The Dutch version of the NPI-Q has been investigated and appears to be a valid instrument [38].

Quality of the relationship: quality of the relationship will be assessed using four items of the University of Southern California Longitudinal Study of ThreeGeneration Families measures of positive affect [39]. The items represent: general closeness, communication, similarity of views on life, and degree of getting along with each other. Answer scales range from 1 (not at all) to 4 (very). Caregivers will answer these items in terms of the current situation and to what extent the relationship changed since illness onset $(1=$ much better, $5=$ much worse). Summed scores will be used as an index of the 
change in relationship quality. A previous study found good internal reliability [40].

Coping: the 44-item Utrechtse Coping List (UCL) will be used to measure seven different coping strategies in the caregiver, including seeking distraction (eight items), expressing emotions (three items), seeking social support (six items), avoiding (eight items), fostering reassuring thoughts (five items), passive coping (seven items), and active coping (seven items). Items are rated on a 4-point scale, ranging from 1 (rarely or never use this strategy) to 4 (very often use this strategy). The reliability and validity has been found sufficient despite some inconsistencies in the literature [41].

Personality: the 12-item Neuroticism domain of the NEO Five-Factor Inventory (NEO-FFI) will be used to identify subjects who are susceptible to psychological distress. This domain measures six traits: anxiety, angry hostility, depression, self-consciousness, impulsiveness, and vulnerability. Item scores will be rated on a 5-point scale, ranging from 0 (strongly disagree) to 4 (strongly agree), and will be accumulated to generate a total sum score (range $0-48$ ). The reliability and internal consistency of the Dutch version of the NEO-FFI is good [42].

\section{Outcome measures in the process evaluation}

As part of the process evaluation, sampling quality and intervention quality will be evaluated to determine the internal and external validity of the intervention program and to reveal facilitators and barriers for the intervention.

Sampling quality: will be evaluated by describing (1) the recruitment and randomization procedure; (2) the informed consent and allocation procedure; and (3) barriers and facilitators to the recruitment of caregivers. Reach will be established by the proportion of caregivers participating and the number of institutions involved in the intervention.

Intervention quality: will be evaluated by determining (1) the relevance of the intervention; (2) the feasibility of the intervention; and (3) the extent to which the intervention was performed according to protocol.

Required data will be collected from the research database, and during the sessions with the coach and the post-intervention assessment. Objective measures of compliance with respect to the ESM procedure (number of completed beeps) and the intervention (number of drop-outs) will be examined. Technical problems with the PsyMate will be logged. Subjective experiences with respect to the intervention (i.e. clearness, relevance, usability, impact on daily functioning, satisfaction with procedure and time burden, suggestions for improvement, and recommendations to other caregivers) will be assessed by means of a quantitative questionnaire and a qualitative interview with the caregiver during the third feedback session.
In addition, subjective experiences with the PsyMate and ESM procedure (e.g. difficulties operating the device, clearness items, readability of the items on the screen, interference with daily activities, and reasons for missed beeps) will be examined.

Performance of the intervention according to protocol will be evaluated with a structured registration form, which includes protocol deviations and duration of each face-to-face session during the intervention period.

\section{Economic evaluation}

The economic evaluation will involve a cost-effectiveness analysis (CEA) and includes the assessment of costs as well as outcomes of the intervention.

Resource use and costs: the Resource Utilization in Dementia - shortened version (RUD-lite) will be used to map the utilization of resources for both the PwD and the caregiver [43]. The RUD-lite assesses both formal and informal resource use, making it possible to calculate costs from a societal point of view. Costs will be calculated by multiplying the quantity of resource use by the cost price per resource unit and will include the period from the baseline assessment until the last follow-up assessment (6 months). All relevant costs will be determined according to the Dutch guidelines for cost calculations in health care [44].

Intervention costs: intervention costs will include the amount of time spent on briefing the caregivers regarding the PsyMate and ESM procedure, the feedback sessions with the coach, administration (e.g. writing short feedback reports), telephone contact, the training session for the coach on how to provide the feedback, and costs of required materials (e.g. PsyMate). The coach will register the duration of the feedback sessions, administration, and telephone contact with the caregiver on a structured registration form. Incremental cost effectiveness ratios (ICER) will be calculated by dividing the difference in total costs between the treatment arms by the difference in average effect size.

Quality of life: caregivers' health related quality of life will be measured with the EuroQol-5D (EQ-5D) and consists of five items representing the following dimensions mobility, self-care, usual activities, pain/discomfort, and anxiety/depression [45]. Respondents indicate their health state by rating each dimension on a 3-level scale, including 1 'no problems', 2 'moderate problems', or 3 'severe problems'. In addition, the EQ-VAS will be used to record caregivers' self-rated health status on a Visual Analogue Scale, anchored at 'the best health you can imagine' (100) and 'the worst health you can imagine' (0). The EuroQol demonstrates a good test-retest reliability [46]. For the cost-effectiveness analysis, quality-adjusted life-years (QALYs) will be calculated according to the Dutch EuroQol tariff [47]. 


\section{Sample size}

The calculation of the sample size will be based on previous studies using the Sense of Competence Questionnaire (SCQ) as outcome measure in intervention studies for caregivers of PwD, the use of repeated measures, withinbetween interaction with a mean effect size of 0.8 [48], and the following assumptions: alpha 0.05, $80 \%$ power and $25 \%$ loss to follow up. Accordingly, we aim to enrol 90 participants in the study (30 participants per group).

\section{Statistical analyses \\ Primary and secondary outcome measures}

Data will be entered twice to guarantee data integrity. Before the analyses, data will be checked for missing values and handled according to their distribution (missing completely at random, missing at random, or not missing at random), and the 'intention-to-treat' principle. Additionally, data will be checked for outliers, and normality. Non-parametric tests will be used in case of non-normality after data transformation. Possible baseline differences in group characteristics will be tested with t-tests for continuous and $x^{2}$-tests for categorical variables.

To investigate changes in the primary and secondary outcomes for each group during the intervention period, an analysis of covariance (ANCOVA) will be performed with outcome at post-intervention as the dependent variable, group (experimental, pseudo-experimental, control) as the between-subject factor, and outcome at baseline and potential confounders (e.g. demographic variables, coping, personality, and quality of the relationship) as covariates. Separate analyses will be conducted for each outcome variable. Group differences in the post-intervention outcome, adjusted for the baseline value, will be examined to test the effectiveness of the intervention. In case group differences are present, the inter-group effect size will be calculated using Cohen's $d$.

To examine changes in the primary and secondary outcomes for each group during the total study period, a linear mixed model (LMM) for repeated measures will be conducted. Analyses will be performed with group as a fixed between-subject factor (3 levels: experimental, pseudo-experimental, and control group) and time as fixed within-subject variable (4) levels: baseline, post-intervention, two-month follow-up, and six-month follow-up) and first-order interactions as additional fixed factors. The LMM will estimate fixed effects (regression slopes) for change in the intervals during (T0-T1) and after (T1-T2, T2-T3) the intervention period. The intervals will be entered as a categorical dummy variable (3 levels). Potential confounders will be added to the model as covariates. Additionally, coach will be added as a random factor to estimate the variability ascribed to the coach.
All data will be analysed using STATA 12.1 [49]. Tests of significance will report mean change and will be two tailed with $\alpha$ set at 0.05 .

\section{ESM outcome measures}

Subjects have to complete at least $33 \%$ of the ESM beeps in order to be included in the ESM data analysis [4]. To investigate changes in momentary positive and negative affect between the 3-day ESM baseline and post measurement for each group, an LMM will be performed for both outcome measures. LMMs are ideally suited for ESM data, since these models take into account the hierarchical data structure in which multiple ESM observations (beep level 1) are nested within days (day level 2), and days are nested within subjects (subject level 3). The XTMIXED command in STATA 12.1 [49] will be used to conduct analyses with group (3 levels: experimental, pseudo-experimental, and control group), time (2 levels: baseline and post-intervention), and the twoway interaction between group and time as fixed factors. Random slopes, representing positive or negative affect, will be added at the subject, day, and beep level. Potential confounders will be included in the model as covariates.

\section{Process evaluation}

Descriptive analyses will be conducted to summarize response rates, overall experiences regarding the ESM intervention and the PsyMate, and additional findings with respect to the sampling and intervention quality.

\section{Confidentiality}

All study-related information will be stored securely at the study site. All participant information will be identified by a coded ID number to maintain participant confidentiality. The principal investigators and project team members will be able to access the data. All records that contain names or other personal identifiers (e.g. informed consent forms) will be stored separately from study records identified by code number. All datasets will be password protected.

\section{Monitoring and participant safety}

The study will be monitored externally by the trial monitoring committee of the MUMC+ (Clinical Trial Center Maastricht). The trial monitoring committee is independent of the study organizers. All adverse events (AEs) and serious adverse events (SAEs) that occur during the study will be recorded during the (feedback) sessions, the post-intervention assessment, and the two- and six-month follow-up assessment. SAEs will be reported to the accredited Medical Ethical Committee that approved the protocol.

\section{Withdrawal of participants}

Participants can leave the study at any time without justification, and without any consequences. 


\section{Discussion}

The current paper presents the study protocol of an RCT to evaluate the effectiveness of an ESM intervention to improve caregivers' sense of competence and control, and psychological well-being. The potential effects of the intervention may help caregivers to keep on caring and prevent them from developing (more) health problems and becoming overburdened. This study contains several unique aspects. To our knowledge, this is the first ESM intervention for caregivers of PwD. ESM might be a promising tool in both research and clinical practice, since it offers the possibility to provide more detailed information on caregiver functioning in the flow of daily life. In this study we will use ESM as an intervention tool, but also as an assessment tool to evaluate the effects of the intervention. Therefore, an important advantage is that the results of this study represent a high level of ecological validity. Another unique aspect is that our intervention focuses on positive rather than negative experiences during the caretaking process. Positive emotions are important facilitators of adaptive coping and might increase caregivers' feelings of being capable to care $[18,50]$. The ESM intervention might enhance caregivers' self-awareness and redirect their behavior to situations that are conducive to positive emotional experiences. Moreover, the intervention includes ESM-derived feedback that is tailored to the caregivers' personal situation and actively involves them in their own empowering process. Both are known to be important aspects of effective caregiver support interventions [14]. The inclusion of a pseudo-experimental condition in our study design allows us to unravel the added value of the ESM-derived feedback to the use of the PsyMate in general. Caregivers might benefit from the use of the PsyMate without receiving any feedback, since repeated self-assessments may lead people to pay more attention to their internal states and own behavior [21]. Lastly, the intervention program will be delivered in daily practice and offers the possibility to be integrated in future clinical practice. The additional process and economic evaluation will provide valuable information on potential facilitators and barriers to implementation, and on the cost-effectiveness of the intervention.

Certain limitations need to be acknowledged in advance. There is a possibility of sample bias. Although participants will be recruited in a wide range of different institutions, the group that agrees to participate in the study might differ from the group that refuses to participate. In general, participants are expected to be relatively young, more highly educated, and more pro-active in seeking for support [51]. Moreover, recent results from our feasibility study, in which caregiver were asked to collect ESM data for only six consecutive days, showed that a large number of caregivers refused to participate because of a too busy time schedule (van Knippenberg et al.: Dealing with daily challenges in dementia (Deal-id study): an innovative approach to assess caregiver functioning in the flow of daily life, submitted). The even more time-intensive nature of the current study might, therefore, cause a selection bias towards caregivers that are not yet facing extreme difficulties in the caregiving process. It is also expected that a number of participants will drop out of the ESM intervention prematurely. In this specific population, it is likely that participants drop out for specific disease related reasons, such as institutionalization or death of the PwD. In a previous ESM study with persons with depression, $15 \%$ of the participants did not complete the 6-week ESM intervention period [9]. The face-to-face sessions with a coach and the delivery of personalized feedback, however, might increase caregivers' motivation to participate given that usual care for dementia caregivers often does not, or very infrequent, include counseling [52].

In conclusion, the results of this study will provide a valuable contribution to the growing knowledge on $\mathrm{m}$ health interventions for caregivers of PwD. Our ESM intervention 'Partner in Sight' is expected to be effective in terms of caregiver well-being and might be an innovative approach to support caregivers of PwD in managing daily challenges during the course of the disease.

\section{Ethics approval and consent to participate}

The Medical Ethical Committee of the MUMC+ approved this study (\#143040). All participants gave informed consent to participate in the study.

\section{Protocol amendments}

Important protocol modifications, which may impact on the conduct of the study or may affect participant safety, including changes in study objectives, study design, study population, sample sizes, study procedures, or significant administrative aspects will require a formal amendment to protocol. Substantial amendments will be notified to the principal investigators and approved by the Medical Ethical Committee of the MUMC+ prior to implementation. Minor changes to the protocol that have no effect on the way the study is conducted will not be notified to the Medical Ethical Committee of the MUMC+, but will be recorded and filed by the investigators.

\section{Consent for publication \\ Not applicable.}

\section{Availability of data}

Not applicable.

\section{Availability of supporting data}

Not applicable.

\section{SPIRIT Guidelines}

This study protocol is based on the SPIRIT 2013 Statement [53]. A SPIRIT checklist is provided as an additional file. 


\section{Appendix}

Table 3 Description of the ESM concepts, items and response choices in the daily, morning and evening questionnaire

\begin{tabular}{|c|c|c|}
\hline \multicolumn{3}{|l|}{ Daily questionnaire } \\
\hline Concept & Item & Rating scale \\
\hline \multirow[t]{4}{*}{ Positive affect } & 1. I feel cheerful & 7-point scale (1 'not at all' to 7 'very much') \\
\hline & 2. I feel relaxed & 7-point scale (1 'not at all' to 7 'very much') \\
\hline & 3. I feel enthusiastic & 7-point scale (1 'not at all' to 7 'very much') \\
\hline & 4. I feel satisfied & 7-point scale (1 'not at all' to 7 'very much') \\
\hline \multirow[t]{8}{*}{ Negative affect } & 5. I feel insecure & 7-point scale (1 'not at all' to 7 'very much') \\
\hline & 6. I feel lonely & 7-point scale (1 'not at all' to 7 'very much') \\
\hline & 7. I feel anxious & 7-point scale (1 'not at all' to 7 'very much') \\
\hline & 8. I feel irritated & 7-point scale (1 'not at all' to 7 'very much') \\
\hline & 9. I feel down & 7-point scale (1 'not at all' to 7 'very much') \\
\hline & 10. I feel desperate & 7-point scale (1 'not at all' to 7 'very much') \\
\hline & 11. I feel confident & 7-point scale (1 'not at all' to 7 'very much') \\
\hline & 12. I feel tensed & -point scale (1 'not at all' to 7 'very much') \\
\hline \multirow[t]{4}{*}{ Self-esteem } & 13. I like myself & 7-point scale (1 'not at all' to 7 'very much') \\
\hline & 14. I am ashamed of myself & 7-point scale (1 'not at all' to 7 'very much') \\
\hline & 15. I doubt myself & 7-point scale (1 'not at all' to 7 'very much') \\
\hline & 16. I am satisfied with myself & 7-point scale (1 'not at all' to 7 'very much') \\
\hline \multirow[t]{4}{*}{ Physical well-being } & 17. I am tired & 7-point scale (1 'not at all' to 7 'very much') \\
\hline & 18. I feel well & 7-point scale (1 'not at all' to 7 'very much') \\
\hline & 19. I am in pain & 7-point scale (1 'not at all' to 7 'very much') \\
\hline & 20. I have problems in walking & 7-point scale (1 'not at all' to 7 'very much') \\
\hline \multirow[t]{9}{*}{ Activity } & 21. What am I doing? (just before the alert) & $\begin{array}{l}\text { Doing nothing; resting; work; household; self care; caring for } \\
\text { partner; active relaxation; passive relaxation; something else }\end{array}$ \\
\hline & 22. And also? & $\begin{array}{l}\text { Doing nothing; resting; work; household; self care; caring for } \\
\text { partner; active relaxation; passive relaxation; something else }\end{array}$ \\
\hline & 23. And...? & $\begin{array}{l}\text { Doing nothing; resting; work; household; self care; caring for } \\
\text { partner; active relaxation; passive relaxation; something else }\end{array}$ \\
\hline & 24. I like doing this & 7-point scale (1 'not at all' to 7 'very much') \\
\hline & 25. I would rather be doing something else & 7-point scale (1 'not at all' to 7 'very much') \\
\hline & 26. This is difficult for me & 7-point scale (1 'not at all' to 7 'very much') \\
\hline & 27. I feel I am being active & 7-point scale (1 'not at all' to 7 'very much') \\
\hline & 28. I can do this well & 7-point scale (1 'not at all' to 7 'very much') \\
\hline & 29. I am doing this activity together with my partner & Yes; no \\
\hline Location & 30. Where am I? & $\begin{array}{l}\text { At home; at family's/friend's place; at work; health care setting; } \\
\text { public place; transport; somewhere else }\end{array}$ \\
\hline \multirow[t]{6}{*}{ Social company } & 31. Who am I with? & $\begin{array}{l}\text { Partner; family; friends; colleagues; health care professional; } \\
\text { acquaintances; strangers/others; nobody }\end{array}$ \\
\hline & 32. With whom else? & $\begin{array}{l}\text { Partner; family; friends; colleagues; health care professional; } \\
\text { acquaintances; strangers/others; nobody }\end{array}$ \\
\hline & 33. And...? & $\begin{array}{l}\text { Partner; family; friends; colleagues; health care professional; } \\
\text { acquaintances; strangers/others; nobody }\end{array}$ \\
\hline & Branching questions in case of being in company: & \\
\hline & 34. I would prefer to be alone & 7-point scale (1 'not at all' to 7 'very much') \\
\hline & 35. I think my company is pleasant & 7-point scale (1 'not at all' to 7 'very much') \\
\hline
\end{tabular}


Table 3 Description of the ESM concepts, items and response choices in the daily, morning and evening questionnaire (Continued)

\begin{tabular}{ll}
\hline 36. I feel at ease in this company \\
Branching questions in case of being alone: \\
34. I would prefer to be in company of others \\
35. I enjoy being alone \\
36. I feel at ease being alone \\
37. Since the last alert the most important thin \\
Events \\
38. How pleasant was this event? \\
39. I had this situation under control \\
40. Was this situation unexpected? \\
41. The event was important to me \\
42. With whom was I? \\
43. This alert disturbed me \\
queneral \\
1. I slept well \\
2. How long did it take before I fell asleep \\
3. How often did I wake up last night \\
4. My partner disturbed my sleep \\
5. I feel rested \\
6. I feel apprehensive about today
\end{tabular}

Evening

questionnaire 7-point scale (1 'not at all' to 7 'very much')

7-point scale (1 'not at all' to 7 'very much')

7-point scale (1 'not at all' to 7 'very much')

7-point scale ( 1 'not at all' to 7 'very much')

(take an event in mind before you continue)

bipolar scale ( -3 'very unpleasant' to +3 'very pleasant')

7 -point scale (1 'not at all' to 7 'very much')

7-point scale (1 'not at all' to 7 'very much')

bipolar scale ( -3 'very unimportant' to +3 'very important')

Partner; nobody; someone else

7-point scale (1 'not at all' to 7 'very much')

7-point scale ( 1 'not at all' to 7 'very much')

0-5 min; 5-15 min; 30-45 min; 45-60 min; 1-2 h; 24 h; >4 h

1 time; 2 times; 3 times; 4 times; 5 times; more than 5 times

7-point scale (1 'not at all' to 7 'very much')

7-point scale (1 'not at all' to 7 'very much')

7-point scale ( 1 'not at all' to 7 'very much')

7-point scale (1 'not at all' to 7 'very much')

7-point scale (1 'not at all' to 7 'very much')

7-point scale (1 'not at all' to 7 'very much')

7-point scale ( 1 'not at all' to 7 'very much')

7-point scale ( 1 'not at all' to 7 'very much')

7 -point scale (1 'not at all' to 7 'very much')

7-point scale (1 'not at all' to 7 'very much')

Visual Analogue Scale (0 'worst imaginable health' to 100 'best imaginable health')

$0 h ; 1 h ; 2 h ; 3 h ; 4 h ; 5 h ;>5 h$ partner today (incl. supervision)

10. Today I felt strained in the interactions with my partner

11. Today I felt stressed due to my care responsibilities

7-point scale ( 1 'not at all' to 7 'very much')

7-point scale (1 'not at all' to 7 'very much')

7-point scale (1 'not at all' to 7 'very much')

2. Today I felt that the situation with my partner did not allow me as much privacy as I would have liked

13. Today I had enough time for myself

7-point scale (1 'not at all' to 7 'very much')

14. Today I was in need of support

15. Today I received enough support

Today, to what extent did your partner suffer from:

16. Being sad or depressed

17. Being anxious our nervous

18. Acting impulsively or embarrassing

19. A loss of interest in activities/other people 7-point scale (1 'not at all' to 7 'very much')

7-point scale ( 1 'not at all' to 7 'very much')

7-point scale (1 'not at all' to 7 'very much')

7-point scale (1 'not at all' to 7 'very much')

7-point scale (1 'not at all' to 7 'very much')

7-point scale (1 'not at all' to 7 'very much') 
Table 3 Description of the ESM concepts, items and response choices in the daily, morning and evening questionnaire (Continued)

\begin{tabular}{|c|c|}
\hline 20. Being irritated or impatient & 7-point scale (1 'not at all' to 7 'very much') \\
\hline 21. Being too cheerful for no reason & 7-point scale ( 1 'not at all' to 7 'very much') \\
\hline 22. Being restless & 7-point scale ( 1 'not at all' to 7 'very much') \\
\hline 23. Agitation/aggression & 7-point scale ( 1 'not at all' to 7 'very much') \\
\hline 24. Beliefs that you know are not true & 7-point scale (1 'not at all' to 7 'very much') \\
\hline 5. Seeing false visions or hearing false voices & 7-point scale (1 'not at all' to 7 'very much') \\
\hline
\end{tabular}

\section{Additional files}

Additional file 1: Recommended items to address in a clinical trial protocol and related documents. (PDF $106 \mathrm{~kb}$ )

\begin{abstract}
Abbreviations
AE: adverse event; ANCOVA: analysis of covariance; CDR: Clinical Dementia Rating scale; CEA: cost-effectiveness analysis; CES-D: Center for Epidemiological Studies Depression Scale; Deal-id: dealing with daily challenges in dementia; EQ-5D: EuroQol-5D; ESM: experience sampling methodology; HADS: Hospital Anxiety and Depression Scale; HIV: Human Immunodeficiency Virus; ICER: incremental cost effectiveness ratios; LMM: linear mixed model; MUMC+: Maastricht University Medical Center+; NEO-FFI: NEO Five-Factor Inventory; NPI: Neuropsychiatric Inventory; NPI-Q: Neuropsychiatric Inventory Questionnaire (NPI-Q); PDA: personal digital assistant; PMS: Pearlin Mastery Scale; PSS: Perceived Stress Scale; PwD: people/persons with dementia; QALY: quality-adjusted life-year; RCT: randomized controlled trial; RUD-lite: Resource Utilization in Dementia - shortened version; SAE: serious adverse event; SCQ: Sense of Competence Questionnaire SSCQ: Short Sense of Competence Questionnaire; UCL: Utrechtse Coping List.
\end{abstract}

\section{Competing interests}

The authors declare that they have no competing interests.

\section{Authors' contributions}

All authors contributed to the design of this study. RvK drafted the manuscript and MdV, FV, RP, and IM provided valuable feedback during the process of writing. All authors read and approved the final manuscript.

\section{Funding}

This study is supported by a grant of the Dutch Alzheimer's Society (Grant nr WE03-2011-06). The grant proposal has been peer-reviewed before the start of the study. The funding body is monitoring the study progress by way of an international scientific forum.

Contact information funding body

Dutch Alzheimer's Society

Contact name: Marco Blom, Manager Scientific Research/Adjunct Director Phone number: 0031306596952

\section{Author details}

${ }^{1}$ Department of Psychiatry and Neuropsychology and Alzheimer Centre Limburg, School for Mental Health and Neurosciences, Maastricht University, Maastricht, The Netherlands. ${ }^{2}$ Department of Neurosciences, Center for Contextual Psychiatry, KU Leuven, Leuven, Belgium.

Received: 11 April 2016 Accepted: 25 April 2016

Published online: 11 May 2016

\section{References}

1. Brodaty H, Donkin M. Family caregivers of people with dementia. Dialogues Clin Neurosci. 2009:11(2):217.

2. Olazarán J, Reisberg B, Clare L, Cruz I, Peña-Casanova J, Del Ser T, Woods B, Beck C, Auer S, Lai C. Nonpharmacological therapies in Alzheimer's disease: a systematic review of efficacy. Dement Geriatr Cogn Disord. 2010;30(2):161-78.
3. Koster EH, De Raedt R, Leyman L, De Lissnyder E. Mood-congruent attention and memory bias in dysphoria: exploring the coherence among information-processing biases. Behav Res Ther. 2010;48(3):219-25.

4. Delespaul PAEG. Assessing schizophrenia in daily life: The experience sampling method. Maastricht: IPSER; 1995.

5. Hektner JM, Schmidt JA, Csikszentmihalyi M. Experience sampling method: Measuring the quality of everyday life. Thousand Oaks, California: Sage Publications Inc.; 2007

6. Myin-Germeys I, Oorschot M, Collip D, Lataster J, Delespaul P, van Os J. Experience sampling research in psychopathology: opening the black box of daily life. Psychol Med. 2009;39(09):1533-47.

7. Shiffman S. Real-time self-report of momentary states in the natural environment: Computerized ecological momentary assessment. In: The science of self-report: Implications for research and practice. Mahwah: Lawrence Erlbaum Associates, Inc.; 2000:277-296.

8. Cain AE, Depp CA, Jeste DV. Ecological momentary assessment in aging research: A critical review. J Psychiatr Res. 2009;43(11):987-96.

9. Kramer I, Simons CJ, Hartmann JA, Menne-Lothmann C, Viechtbauer W, Peeters F, Schruers K, Bemmel AL, Myin-Germeys I, Delespaul P. A therapeutic application of the experience sampling method in the treatment of depression: a randomized controlled trial. World Psychiatry. 2014;13(1):68-77.

10. Myin-Germeys I, Birchwood M, Kwapil T. From environment to therapy in psychosis: a real-world momentary assessment approach. Schizophr Bull. 2011;37(2):244-7.

11. Schmidt U, Landau S, Pombo-Carril MG, Bara-Carril N, Reid Y, Murray K, Treasure J, Katzman M. Does personalized feedback improve the outcome of cognitive-behavioural guided self-care in bulimia nervosa? A preliminary randomized controlled trial. Br J Clin Psychol. 2006;45(1):111-21.

12. Smits JA, Powers MB, Buxkamper R, Telch MJ. The efficacy of videotape feedback for enhancing the effects of exposure-based treatment for social anxiety disorder: a controlled investigation. Behav Res Ther. 2006;44(12): 1773-85.

13. Wichers M, Simons C, Kramer I, Hartmann J, Lothmann C, Myin-Germeys I, van Bemmel A, Peeters F, Delespaul P, van Os J. Momentary assessment technology as a tool to help patients with depression help themselves. Acta Psychiatr Scand. 2011;124(4):262-72.

14. Pinquart M, Sörensen S. Helping caregivers of persons with dementia: which interventions work and how large are their effects? Int Psychogeriatr. 2006;18(04):577-95.

15. Boots L, Vugt M, Knippenberg R, Kempen G, Verhey F. A systematic review of Internet-based supportive interventions for caregivers of patients with dementia. Int J Geriatr Psychiatry. 2014:29(4):331-44.

16. Boots LM, Wolfs CA, Verhey FR, Kempen Gl, de Vugt ME. Qualitative study on needs and wishes of early-stage dementia caregivers: the paradox between needing and accepting help. Int Psychogeriatr. 2015;27(06):927-36.

17. Fredrickson BL. What good are positive emotions? Rev Gen Psychol. 1998; 2(3):300.

18. de Vugt ME, Stevens F, Aalten P, Lousberg R, Jaspers N, Winkens I, Jolles J, Verhey FR. Do caregiver management strategies influence patient behaviour in dementia? Int J Geriatr Psychiatry. 2004;19(1):85-92.

19. Fredrickson $\mathrm{BL}$, Joiner T. Positive emotions trigger upward spirals toward emotional well-being. Psychol Sci. 2002;13(2):172-5.

20. de Vugt ME, Verhey FR. The impact of early dementia diagnosis and intervention on informal caregivers. Prog Neurobiol. 2013;110:54-62.

21. Ben-Zeev D, Young MA, Madsen JW. Retrospective recall of affect in clinically depressed individuals and controls. Cogn Emot. 2009;23(5):1021-40. 
22. Fonareva I, Amen AM, Ellingson RM, Oken BS. Differences in stress-related ratings between research center and home environments in dementia caregivers using ecological momentary assessment. Int Psychogeriatr. 2012; 24(01):90-8.

23. Poulin MJ, Brown SL, Ubel PA, Smith DM, Jankovic A, Langa KM. Does a helping hand mean a heavy heart? Helping behavior and well-being among spouse caregivers. Psychol Aging. 2010;25(1):108.

24. Leontjevas R, Gerritsen DL, Koopmans RT, Smalbrugge M, Vernooij-Dassen MJ. Process evaluation to explore internal and external validity of the "Act in Case of Depression" care program in nursing homes. J Am Med Dir Assoc. 2012;13(5):488. e481-488. e488.

25. Oorschot M, Lataster T, Thewissen V, Wichers M, Myin-Germeys I. Mobile assessment in schizophrenia: a data-driven momentary approach. Schizophr Bull. 2012;38(3):405-13

26. Myin-Germeys I, Krabbendam L, Jolles J, Delespaul PA, van Os J. Are cognitive impairments associated with sensitivity to stress in schizophrenia? An experience sampling study. Am J Psychiatry. 2002;159(3):443-9.

27. Palmier-Claus JE, Myin-Germeys I, Barkus E, Bentley L, Udachina A, Delespaul P, Lewis SW, Dunn G. Experience sampling research in individuals with mental illness: reflections and guidance. Acta Psychiatr Scand. 2011;123(1):12-20.

28. Vernooij-Dassen MJ, Felling AJ, Brummelkamp E, Dauzenberg MG, Bos GA, Grol R. Assessment of caregiver's competence in dealing with the burden of caregiving for a dementia patient: a Short Sense of Competence Questionnaire (SSCQ) suitable for clinical practice. J Am Geriatr Soc. 1999;47(2):256-7.

29. Pearlin LI, Schooler C. The structure of coping. J Health Soc Behav. 1978; 19(1):2-21.

30. Kempen Gl. Psychometric Properties of GLAS Baseline Measures: a Pilot Study (in Dutch). The Netherlands: Northern Centre for Healthcare Research, University of Groningen; 1992.

31. Radloff LS. The CES-D scale a self-report depression scale for research in the general population. Appl Psychol Meas. 1977;1(3):385-401.

32. Cohen S, Kamarck T, Mermelstein R. A global measure of perceived stress. J Health Soc Behav. 1983;24(4):385-396.

33. Zigmond AS, Snaith RP. The hospital anxiety and depression scale. Acta Psychiatr Scand. 1983;67(6):361-70

34. Bjelland I, Dahl AA, Haug T, Neckelmann D. The validity of the Hospital Anxiety and Depression Scale: an updated literature review. J Psychosom Res. 2002;52(2):69-77.

35. Hughes CP, Berg L, Danziger WL, Coben LA, Martin RL. A new clinical scale for the staging of dementia. Br J Psychiatry. 1982;140(6):566-72.

36. Morris JC. Clinical dementia rating: a reliable and valid diagnostic and staging measure for dementia of the Alzheimer type. Int Psychogeriatr. 1997;9(S1):173-6.

37. Cummings $J$ L. The Neuropsychiatric Inventory Assessing psychopathology in dementia patients. Neurology. 1997;48(5 Suppl 6):10S-6.

38. De Jonghe J, Kat MG, Kalisvaart C, Boelaarts L. Neuropsychiatric inventory questionnaire (NPI-Q): A validity study of the Dutch form. Tijdschr Geronto Geriatr. 2003;34(2):74-7.

39. Lawrence RH, Tennstedt SL, Assmann SF. Quality of the caregiver-care recipient relationship: Does it offset negative consequences of caregiving for family caregivers? Psychol Aging. 1998;13(1):150.

40. De Vugt ME, Stevens F, Aalten P, Lousberg R, Jaspers N, Winkens I, Jolles J, Verhey FR. Behavioural disturbances in dementia patients and quality of the marital relationship. Int J Geriatr Psychiatry. 2003;18(2):149-54.

41. Schreurs P, Willige Van De G, Brosschot JF, Tellegen B, Graus GMH. De Utrechtse coping lijst: UCL. Omgaan met problemen en gebeurtenissen, herziene handleiding.[the Utrecht's coping list: UCL. Managing problems and events, revised manual]. Lisse: Swets Test Publishers; 1993.

42. Hoekstra H, Ormel J, De Fruyt F. Handleiding NEO persoonlijkheidsvragenlijsten [Manual NEO personality questionnaires]. Lisse: Swets Test Services; 1996.

43. Wimo A, Winblad B, Stoffler A, Wirth Y, Mobius HJ. Resource utilisation and cost analysis of memantine in patients with moderate to severe Alzheimer's disease. Pharmacoeconomics. 2003;21(5):327-40.

44. Hakkaart-van Roijen L, Tan S, Bouwmans C. Handleiding voor kostenonderzoek: Methoden en standaard kostprijzen voor economische evaluaties in de gezondheidszorg. Diemen, The Netherlands: College voor zorgverzekeringen; 2010.

45. Brooks R, Group E. EuroQol: the current state of play. Health Policy. 1996; 37(1):53-72.
46. van Agt HM, Essink-Bot M-L, Krabbe PF, Bonsel GJ. Test-retest reliability of health state valuations collected with the EuroQol questionnaire. Soc Sci Med. 1994;39(11):1537-44.

47. Lamers L, Stalmeier P, McDonnell J, Krabbe P, van Busschbach J. [Measuring the quality of life in economic evaluations: the Dutch EQ-5D tariff]. Ned Tijdschr Geneeskd. 2005;149(28):1574-8.

48. Graff MJ, Vernooij-Dassen MJ, Thijssen M, Dekker J, Hoefnagels WH, Rikkert MGO. Community based occupational therapy for patients with dementia and their care givers: randomised controlled trial. BMJ. 2006:333(7580):1196.

49. Stata S. Release 12. Statistical Software. College Station: StataCorp LP; 2011.

50. Folkman S, Moskowitz JT. Positive affect and the other side of coping. Am Psychol. 2000;55(6):647.

51. Andersson G, Bergström J, Holländare F, Carlbring P, Kaldo V, Ekselius L. Internet-based self-help for depression: randomised controlled trial. $\mathrm{Br}$ J Psychiatry. 2005;187(5):456-61.

52. Campbell M, Fitzpatrick R, Haines A, Kinmonth AL. Framework for design and evaluation of complex interventions to improve health. Br Med J. 2000; 321(7262):694.

53. Chan A-W, Tetzlaff JM, Gøtzsche PC, Altman DG, Mann H, Berlin JA, Dickersin K, Hróbjartsson A, Schulz KF, Parulekar WR. SPIRIT 2013 explanation and elaboration: guidance for protocols of clinical trials. BMJ. 2013;346: e7586.

\section{Submit your next manuscript to BioMed Central and we will help you at every step:}

- We accept pre-submission inquiries

- Our selector tool helps you to find the most relevant journal

- We provide round the clock customer support

- Convenient online submission

- Thorough peer review

- Inclusion in PubMed and all major indexing services

- Maximum visibility for your research

Submit your manuscript at www.biomedcentral.com/submit

) Biomed Central 